\title{
PERFIL DE PACIENTES ATENDIDOS POR TENTATIVA DE SUICÍDIO EM UM CENTRO DE ASSISTÊNCIA TOXICOLÓGICA ${ }^{1}$
}

\author{
PROFILE OF PATIENTS TREATED FOR ATTEMPTED SUICIDE \\ IN TOXICOLOGY ASSISTANCE CENTER
}

\section{PERFIL DE LOS PACIENTES TRATADOS POR INTENTO DE SUICIDIO EN UN CENTRO DE ATENCIÓN TOXICOLÓGICA}

\author{
DAIANe Luz MOREIRA* \\ Mariana CaValcante Martins ${ }^{* *}$ \\ FABIANE do AMARAL GUBERT ${ }^{* * *}$ \\ Fernando Sérgio Pereira de Sousa ${ }^{* * *}$
}

\begin{abstract}
RESUMO
Objetivo: Caracterizar os pacientes atendidos por tentativa de suicídio, no Centro de Assistência Toxicológica de um hospital de Fortaleza, Ceará, Brasil. Material e método: Estudo retrospectivo realizado com 409 Fichas de Notificação e de Atendimento dos pacientes atendidos em um Centro de Assistência Toxicológica no ano de 2010. A análise se deu por meio de comparação entre proporções, seguindo o nível de significância de 5\%. O estudo foi aprovado pelo Comitê de Ética em Pesquisa. Resultados: Evidenciou-se a predominância do ato suicida no sexo feminino $(58,2 \%)$ e nos adultos jovens $(62,8 \%)$, procedentes da zona urbana $(87,8 \%)$ e estudantes $(16,1 \%)$ em relação à ocupação. As tentativas de suicídio ocorreram na própria residência do indivíduo $(89,5 \%)$, utilizando a autointoxicação por medicamentos (51,8\%), seguidos pelos agrotóxicos de uso agrícola $(31,2 \%)$. Foram identificados sete casos $(1,7 \%)$ em que os pacientes possuíam transtorno mental. O tratamento dos pacientes atendidos por tentativa de suicídio foi baseado em sua maioria na assistência clínica (98,8\%), sem suporte psicológico ou psiquiátrico durante o período de internação ou encaminhamento para instituição psiquiátrica após a melhora do quadro clínico da intoxicação. Identificou-se que houve uma forte relação entre o grau de envenenamento dos pacientes e a variável sexo $(\mathrm{p}<0,001)$. Conclusão: Percebe-se a necessidade de se desempenhar uma maior integralidade na assistência aos indivíduos que tentam suicídio, realizando ações de promoção e prevenção, uma vez que esse agravo pode e deve ser evitado.
\end{abstract}

Palavras chave: Enfermagem psiquiátrica, tentativa de suicídio, assistência toxicológica.

\footnotetext{
${ }^{1}$ É um destaque da Monografia de Graduação em Enfermagem: Perfil de pacientes atendidos por tentativa de suicídio no setor de emergência de um hospital de Fortaleza - Ceará, 2010. Fortaleza, Faculdade Integrada da Grande Fortaleza, 2011. *Enfermeira, especialista em Saúde da Família pela Universidade Federal do Ceará, Fortaleza-CE, Brasil. E-mail: daianeluz@ hotmail.com

${ }^{* *}$ Enfermeira, Doutora em Enfermagem pela Universidade Federal do Ceará (UFC), Docente do Curso de Graduação em Enfermagem da UFC. Fortaleza, Ceará, Brasil. E-mail: marianaenfermagem@hotmail.com

${ }_{* * *}$ Enfermeira. Doutora em Enfermagem pela Universidade Federal do Ceará (UFC). Docente do Curso de Graduação em Enfermagem da UFC. Fortaleza, Ceará, Brasil. E-mail: fabianegubert@hotmail.com

${ }^{* * * *}$ Enfermeiro. Mestre em Saúde Pública pela Universidade Estadual do Ceará (UECE). Docente do Curso de Graduação em Enfermagem da Universidade Federal do Piauí (UFPI). Floriano, Piauí. Brasil. E-mail: fernando_sergio_1@hotmail.com
} 


\begin{abstract}
Objective: This study aimed to characterize the patients treated for attempted suicide at the Center for Toxicological Assistance a hospital in Fortaleza, Ceará, Brazil. Method: Retrospective study of 409 Sheets Notification and Call of patients attended in a Toxicological Assistance Center in 2010. The analysis was done by comparing proportions, following the significance level of $5 \%$. The study was approved by the Research Ethics Committee. Results: Revealed the prevalence of suicide attempt among females $(58.2 \%)$ and young adults $(62.8 \%)$, coming from urban areas $(87.8 \%)$, students $(16.1 \%)$ compared to the occupation. Suicide attempts occurred in the individual's own residence $(89.5 \%)$, autointoxication by using the medications (51.8\%), followed by agricultural pesticides (31.2\%). We identified seven cases (1.7\%) in which patients had mental disorder. Treatment of patients treated for attempted suicide was based mostly on clinical care (98.8\%), with no psychological or psychiatric support during hospitalization or referral to a psychiatric institution after clinical improvement of intoxication. It was identified that there was a strong relationship between the degree of poisoning of patients and gender $(\mathrm{p}<0.001)$. Conclusion: There is the need to perform a more comprehensive assistance to individuals who attempt suicide, conducting promotion and prevention, since this injury can and should be avoided.
\end{abstract}

Key words: Psychiatric nursing, attempt to suicide, toxicological assistance.

\title{
RESUMEN
}

Objetivo: Caracterizar pacientes asistidos por intento de suicidio en el Centro de Atención Toxicológica de un hospital de Fortaleza, Ceará, Brasil. Material y método: Estudio retrospectivo, con 409 fichas de notificación y llamadas de pacientes atendidos en un Centro de Atención Toxicológica en 2010. El análisis se realizó mediante comparación de proporciones, siguiendo el nivel de significación de 5\%. Estudio aprobado por el Comité de Ética en Investigación. Resultados: Predominio de acto suicida en el sexo femenino (58,2\%) y en adultos jóvenes $(62,8 \%)$, procedentes de zonas urbanas $(87,8 \%)$ y estudiantes $(16,1 \%)$ en relación a la ocupación. Los intentos de suicidio ocurrieron en la casa de la persona $(89,5 \%)$, utilizándose autointoxicación por medicamentos $(51,8 \%)$, seguidos por pesticidas agrícolas $(31,2 \%)$. Fueron identificados siete casos $(1,7 \%)$ cuyos pacientes poseían trastorno mental. El tratamiento de los pacientes asistidos por intento de suicidio fue basado en su mayoría en la atención clínica $(98,8 \%)$, sin apoyo psicológico o psiquiátrico durante el período de hospitalización o encaminamiento a la institución mental después de la mejora del cuadro clínico de la intoxicación. Hubo fuerte relación entre el grado de envenenamiento de los pacientes y la variable sexo $(\mathrm{p}<0,001)$. Conclusión: Hay necesidad de desarrollar mayor atención integral a personas que intentan suicidarse, realizándose acciones de promoción y prevención, ya que este daño puede y debe evitarse.

Palabras clave: Enfermería psiquiátrica, intento de suicidio, atención toxicológica.

Fecha recepción: 29/04/14 Fecha aceptación: 16/06/15

\section{INTRODUÇÃO}

As mortes por suicídio representam um grande problema de saúde pública. Em todo o mundo e em números absolutos, os suicídios matam mais que os homicídios e as guerras juntos (1).

Suicídio é toda morte causada pela ação da própria vítima, tendo esta conhecimento dos resultados de seu ato, já a tentativa de suicídio é o ato consciente e não efetivado de autodestruição (2).

A Organização Mundial de Saúde consi- 
dera o suicídio um problema de saúde pública, visto que dados apontam que uma média de quase um milhão de pessoas se suicida a cada ano. Além disso, durante os últimos 45 anos, as taxas mundiais de suicídio aumentaram $60 \%$ (3).

No Brasil, os suicídios representam 0,8\% do total de óbitos e 6,6\% das mortes por causas externas, ficando na terceira posição no conjunto das mortes por causas externas. No entanto, no período de 2000 a 2009, o risco de morte por acidentes e por causas violentas apresentou um aumento discreto de 4,9 e $4,4 \%$, respectivamente, e o risco de morte por suicídios teve um crescimento de $22,5 \%$ (4). Segundo um estudo descritivo (5) realizado no período entre 1980 e 2006 a taxa de mortalidade nacional brasileira para suicídio teve um aumento de $29,5 \%$, indo de 4,4 para 5,7 mortes por 100.000 habitantes.

Alguns estados ultrapassam a taxa de mortalidade nacional, o Ceará está entre eles e, no último boletim lançado pela Secretaria de Saúde do Estado, apresentou uma taxa de 6,6 por 100.000 habitantes no ano de 2005, estando Fortaleza, que é a capital, a um décimo a mais que isso. Considerando que o estado do Ceará e a cidade de Fortaleza possuem curvas de mortalidade ascendente para o suicídio, hoje esses números devem estar bem maiores. O que ainda não pode ser verificado pela falta de informações recentes (6).

Mesmo assim, o Brasil é um dos países que possui baixa taxa de mortalidade por suicídio, no entanto, por ser populoso, faz parte do quadro de países de maiores números absolutos. Tendo em vista que o Brasil é um país muito grande, existem especificidades quanto ao suicídio em suas diversas regiões e conhecer as diferenças regionais através de análises epidemiológicas populacionais é importante para o desenvolvimento de efetivas políticas públicas de prevenção do suicídio.

$\mathrm{O}$ fenômeno suicídio não merece uma consideração simplificada e evidente, significando apenas uma atração pela morte como tem sido entendido ou como um sinônimo de morte. O suicídio pode emergir de uma série de envolvimentos intrínsecos a uma pessoa e o seu contexto de vida em determinado momento, podendo manifestar-se não só em um ato suicida, mas em tentativas de suicídio e em ideações suicidas (7).

Em razão da não abordagem do suicídio em uma conjuntura mais ampla, considerando sua forma implícita e indissociável com a ideia de morte, um certo tabu tem sido criado em torno do mesmo, evidenciado pelo descaso e pelo comportamento das pessoas que não pensam seriamente no suicídio, como se preferissem acreditar que este ato, de fato, não ocorresse. Assim, ele tem sido aceito enquanto fantasia, tendo lugar prioritário na ficção (7).

Assim, deve-se levar em consideração três componentes básicos inerentes na decisão de uma pessoa que está envolvida no comportamento suicida: a) um nível emocional, intenso sofrimento; b) um nível comportamental, a falta de recursos psicológicos para lidar; c) um nível cognitivo, um desespero profundo sobre o futuro, acompanhada da percepção da morte como a única solução (8).

Não somentes os aspectos psicológicos, mas os aspectos epidemiológicos tem ajudado a evitar o suicidio, tais como: abordagem social -renda, situação conjugal, escolaridade, etc; demográfica -moradia, locazição, dentre outros; clinicas - meio utilizado para tentativa, local do ato (1); essses aspectos devem ser investigados, com a finalidade de traçar o perfil e atuar de forma mais direcionada com esse pacientes de forma preventiva (9).

Então, julga-se pertinente conhecer o perfil desses pacientes que tentaram suicídio, como sendo um ponto inicial par atuar na prevenção no estado do Ceará, diante da escasses de estudos que envolvem essa temática. Ressalta-se que a maioria das pesquisas científicas referentes à epidemiologia dos comportamentos suicidas (suicídio, tentativa de suicídio e ideação suicida) está concentrada no sul e no sudeste do Brasil. Para tanto, com a realização do estudo, espera-se contri- 
buir para a ampliação das discussões sobre essa problemática e colaborar para a construção de uma prática que valorize e fortaleça a abordagem multidisciplinar e interdisciplinar frente ao suicídio de forma preventiva e direcionada.

Assim o objetivo do estudo é caracterizar os pacientes atendidos por tentativa de suicídio, no Centro de Assistência Toxicológica de um hospital de Fortaleza, Ceará, Brasil.

\section{MATERIAL E MÉTODO}

Estudo do tipo documental, descritivo e retrospectivo com abordagem quantitativa. Os dados foram coletados a partir das fichas de notificação e de atendimento dos pacientes diagnosticado por tentativa de suicídio conforme o Código Internacional de Doenças (CID 10).

A amostra da pesquisa foi todo o universo de 409 pacientes atendidos em Centro de Assistência Toxicológica (CEATOX) de um hospital público de alta complexidade, referencia em urgência, emergência e trauma, localizado em Fortaleza, Ceará.

$\mathrm{O}$ instrumento utilizado para a coleta de dados foi um formulário construído a partir da ficha de notificação e de atendimento do CEATOX, contemplando as seguintes variáveis: identificação -nome, sexo, data de nascimento, estado civil, procedência e profissão; formas utilizadas pelo paciente para a tentativa de suicídio -local de ocorrência, tipo de agente tóxico, via de exposição, se utilizou mais de uma toxina; bem como os possíveis motivos, como presença de transtorno mental; além dos aspectos clínicos e de assistência ao paciente tais como, avaliação, internação, condutas e encaminhamentos, e evolução.

A organização e o processamento dos dados foram realizados pelo programa estatístico Statistical Package for the Social Sciences, versão 19.0, sendo estabelecido nível de signi- ficância de 5\% ( $\mathrm{p}<0,05)$. A variável, nível de envenenamento dos pacientes, foi escolhida para comparação entre proporções por meio do qui-quadrado $\left(\chi^{2}\right)$. Os resultados foram apresentados em forma de tabelas no intuito de facilitar a apresentação e a compreensão dos dados.

A pesquisa seguiu os preceitos da Resolução 466/2012 do Conselho Nacional de Saúde, que trata da ética em pesquisas que envolvem humanos. Por tratar-se de uma pesquisa que utiliza dados secundários (envolve indiretamente seres humanos), o pesquisador assinou o Termo de Fiel Depositário, onde assumiu o compromisso de seguir as imposições da resolução já mencionada, mantendo a confidencialidade e a privacidade das informações obtidas através das fichas de notificação e de atendimento dos participantes do estudo, além de garantir justiça e equidade no uso dessas informações. Também foi solicitada autorização da Coordenação do CEATOX da instituição hospitalar, que permitiu a realização do estudo, por meio do Termo de Anuência. O projeto foi submetido à análise do Comitê de Ética em Pesquisa (CEP) da Academia Cearense de Odontologia, tendo parecer favorável sob processo No 315.

\section{RESULTADOS}

Considerando-se as características sociodemográficas dos pacientes, observou-se que 238 pertenciam ao sexo feminino $(58,2 \%)$ e que se encontravam na faixa etária entre 20 a 40 anos (62,8\%), seguido da população maior que 40 anos (20,1\%), percebendo-se, assim, a maior predominância do ato suicida no sexo feminino e nos adultos jovens (Tabela 1).

Também foram colhidas informações quanto à ocupação dos pacientes, sendo observado que houve um grande número de casos em que a profissão foi ignorada $(28,2 \%)$. 
As categorias que tiveram a maior prevalência foram às compostas por: estudantes $(16,1 \%)$, outras profissões diversificadas também abrangeram uma boa parte da população $(15,4 \%)$, desempregados (14,9\%), empregadas domésticas $(6,8 \%)$ e agriculto- res $(6,6 \%)$. Dando seguimento, percebe-se que quanto ao local de procedência, a maior parte dos indivíduos residia em zona urbana $(87,8 \%)$, e uma pequena parcela foi ignorada $(0,7 \%)$. Pode-se observar esses resultados pela Tabela 1.

Tabela 1. Caracterização dos pacientes que tentaram suicídio no ano de 2010, $n=409$. Fortaleza-CE, 2011.

\begin{tabular}{|c|c|c|}
\hline Características & $\mathbf{N}$ & $\%$ \\
\hline \multicolumn{3}{|l|}{ Sexo } \\
\hline Masculino & 171 & 41,8 \\
\hline Feminino & 238 & 58,2 \\
\hline Total & 409 & 100 \\
\hline \multicolumn{3}{|l|}{ Idade } \\
\hline 10 a 19 anos & 70 & 17,1 \\
\hline 20 a 40 anos & 257 & 62,8 \\
\hline 41 a 60 anos & 71 & 17,4 \\
\hline Maior de 60 anos & 11 & 2,7 \\
\hline Total & 409 & 100 \\
\hline \multicolumn{3}{|l|}{ Ocupação } \\
\hline Estudante & 66 & 16,1 \\
\hline Agricultor & 27 & 6,6 \\
\hline Vendedor & 5 & 1,2 \\
\hline Comerciante & 7 & 1,7 \\
\hline Empregada Doméstica & 28 & 6,8 \\
\hline Dona de Casa & 6 & 1,5 \\
\hline Serviços Gerais & 4 & 1,1 \\
\hline Construção e Marcenaria & 12 & 2,9 \\
\hline Culinária & 5 & 1,2 \\
\hline Desempregado & 61 & 14,9 \\
\hline Aposentado & 10 & 2,4 \\
\hline Outros & 63 & 15,4 \\
\hline Ignorado & 115 & 28,2 \\
\hline Total & 409 & 100 \\
\hline \multicolumn{3}{|l|}{ Local de Procedência } \\
\hline Zona Urbana & 359 & 87,8 \\
\hline Zona Rural & 47 & 11,5 \\
\hline Ignorado & 3 & 0,7 \\
\hline Total & 409 & 100 \\
\hline
\end{tabular}


Com relação ao local de ocorrência ( $\mathrm{Ta}$ bela 2) da tentativa de suicídio, a maior parte da população realizou o ato na própria residência $(89,5 \%)$, a segunda proporção mais significativa foi ignorada $(4,4 \%)$, e em seguida o ambiente externo (3,7\%). Outros locais também foram selecionados pelos indivíduos, assim compreendidos: ambiente de trabalho $(1 \%)$, trajeto de trabalho $(0,5 \%)$ e escola $(0,5 \%)$. Diante desses dados, entende-se que o local em que os pacientes obtiveram maior acesso às toxinas foi no próprio lar.

Quanto ao uso desses agentes tóxicos, apesar de terem sido identificados 409 casos de tentativas de suicídio, houve 561 ocorrências quanto à utilização de substâncias tóxicas, devido ao fato de 121 pacientes $(29,6 \%)$ usaram mais de um agente tóxico na ação suicida. Dentre os tóxicos mais utilizados, no intuito de findar a própria vida, estão os medicamentos, que compreenderam 51,8\% de todas as substâncias que foram empregadas pela população em questão. Conforme o apresentado na Tabela 2, houve 55 ocorrências do uso de anticonvulsivantes (9,9\%) na população geral, 41 de antidepressivos (7,3\%), 37 de ansiolíticos (6,6\%), e 31 de antipsicóticos (5,5\%).

Tabela 2. Características em relação ao local de ocorrência, tóxico utilizado e existência de transtorno mental. Fortaleza-CE, 2011.

\begin{tabular}{lrc}
\hline Características & N & $\%$ \\
\hline Local de Ocorrência & & \\
\hline Residência & 366 & 89,5 \\
Ambiente de Trabalho & 4 & 1 \\
Trajeto de Trabalho & 2 & 0,5 \\
Escola & 2 & 0,5 \\
Ambiente Externo & 15 & 3,7 \\
Ignorado & 18 & 4,4 \\
Outros & 2 & 0,4 \\
\hline Total & 409 & 100 \\
\hline Tóxico Utilizado & & \\
\hline Chumbinho & 113 & 20,1 \\
Carbamato & 23 & 4 \\
Organofosforado & 27 & 4,8 \\
Outro Agrotóxico/ Uso Agrícola & 13 & 2,3 \\
Ácido Muriático & 7 & 1,2 \\
Outro Domissanitário & 10 & 1,8 \\
Antidepressivo & 41 & 7,3 \\
Anticonvulsivante & 55 & 9,9 \\
Ansiolítico & 37 & 6,6 \\
Antipsicótico & 31 & 5,5 \\
Agente Tóxico Desconhecido & 4 & 0,7 \\
Outros Medicamentos & 126 & 22,5 \\
Produto Veterinário & 16 & 2,9 \\
\hline
\end{tabular}




\begin{tabular}{lrc}
\hline Raticida & 27 & 4,8 \\
Cosmético & 2 & 0,4 \\
Produto Químico Industrial & 5 & 0,9 \\
Droga de Abuso & 17 & 3 \\
Agrotóxico/ Uso Doméstico & 7 & 1,3 \\
\hline Total & 561 & 100 \\
\hline Mais de um Agente Tóxico & & \\
\hline Sim & 121 & 29,6 \\
Não & 288 & 70,4 \\
\hline Total & 409 & 100 \\
\hline Transtorno Mental & & \\
\hline Sim & 7 & 1,7 \\
Não & 402 & 98,3 \\
\hline Total & 409 & 100 \\
\hline
\end{tabular}

Outro grupo de agentes tóxicos que obteve grande representatividade, conforme a Tabela 2 foi o dos agrotóxicos de uso agrícola $(31,2 \%)$, em que, no total das ocorrências, houve 113 casos nos quais os indivíduos se utilizaram de "chumbinho" (20,1\%), 27 casos de organofosforados $(4,8 \%), 23$ de carbamatos não especificados (4\%), e outros (2,3\%). Em seguida, os demais agrupamentos de substâncias tóxicas compuseram os $17 \%$ restantes do total das ocorrências e incluem: os raticidas $(4,8 \%)$, as drogas de abuso (3\%), os domissanitários (3\%), os produtos veterinários $(2,9 \%)$, os agrotóxicos de uso doméstico $(1,2 \%)$, os produtos químicos industriais $(0,9 \%)$, os agentes tóxicos desconhecidos $(0,7 \%)$ e os cosméticos $(0,4 \%)$.

Das 409 fichas de notificação do CEATOX por tentativa de suicídio no ano de 2010, puderam ser identificados sete casos de pacientes com transtorno mental $(1,7 \%)$, conforme a Tabela 2.

A Tabela 3 evidencia que na quase totalidade da população estudada, a via mais utilizada de exposição ao agente tóxico foi a oral $(99,3 \%)$, tendo a principal conduta utilizada no tratamento a associação de lavagem gás- trica com administração de carvão ativado $(64,3 \%)$, seguida de utilização isolada da administração de carvão ativado (12,2\%), outras condutas $(11,9 \%)$ e realização isolada de lavagem gástrica $(6,4 \%)$. Ressalta-se que dos pacientes que foram atendidos, $307(75,1 \%)$ foram internados para a realização de tratamento.

Com relação à evolução do quadro dos pacientes (Tabela 3), 254 casos (62,1\%) evoluíram para estabilização do quadro de intoxicação, $68(16,6 \%)$ para cura não confirmada, 58 (14,2\%) foram transferido para outra instituição hospitalar, $23(5,6 \%)$ foram ignorados, três $(0,7 \%)$ evadiram-se e um $(0,2 \%)$ assinou o Termo de Alta.

Verificou-se que o nível de envenenamento dos pacientes associou-se significativamente com a variável sexo $(p<0,001)$. De acordo com os dados delineados na Tabela 4, a maior parte da população feminina foi avaliada como estado de envenenamento moderado $(32,7 \%)$. Na população masculina, a maior proporção dos indivíduos foi avaliada como estado de envenenamento grave $(36,3 \%)$. Conforme pode ser verificado na Tabela 4. 
Tabela 3. Caracterização da via de administração, internação, principais condutas e evolução do quadro. Fortaleza, 2011.

\begin{tabular}{|c|c|c|}
\hline Características & $\mathbf{N}$ & $\%$ \\
\hline \multicolumn{3}{|l|}{ Via de Exposição } \\
\hline Oral & 406 & 99,3 \\
\hline Respiratória & 2 & 0,5 \\
\hline Parenteral & 1 & 0,2 \\
\hline Total & 409 & 100 \\
\hline \multicolumn{3}{|l|}{ Internação } \\
\hline $\operatorname{Sim}$ & 307 & 75 \\
\hline Não & 94 & 23 \\
\hline Ignorado & 8 & 2 \\
\hline Total & 409 & 100 \\
\hline \multicolumn{3}{|l|}{ Principais Condutas e Encaminhamentos } \\
\hline Observação Clínica & 4 & 1 \\
\hline Tratamento Sintomático & 6 & 1,5 \\
\hline Tratamento de Suporte & 2 & 0,5 \\
\hline Descontaminação Cutâneo Mucosa & 1 & 0,2 \\
\hline Lavagem Gástrica & 26 & 6,4 \\
\hline Lavagem Intestinal & 1 & 0,2 \\
\hline Suporte Psicológico ou Psiquiátrico & 4 & 1 \\
\hline Carvão Ativado & 50 & 12,2 \\
\hline Uso de Antídoto & 2 & 0,5 \\
\hline Encaminhamento para Instituição Psiquiátrica & 1 & 0,2 \\
\hline Lavagem Gástrica e Carvão Ativado & 263 & 64,3 \\
\hline Outros & 49 & 12 \\
\hline Total & 409 & 100 \\
\hline \multicolumn{3}{|l|}{ Evolução } \\
\hline Melhora Clínica & 254 & 62,1 \\
\hline Melhora Clínica não confirmada & 68 & 16,6 \\
\hline Sequela & 2 & 0,5 \\
\hline Transferência para Outra Instituição Hospitalar & 58 & 5,6 \\
\hline Evasão & 3 & 14,2 \\
\hline Assinatura de Termo de Alta & 1 & 0,7 \\
\hline Ignorado & 23 & 0,3 \\
\hline Total & 409 & 100 \\
\hline
\end{tabular}


Tabela 4. Relação do nível de envenenamento por sexo, dos pacientes que tentaram suicídio no ano de 2010. Fortaleza-CE, 2011.

\begin{tabular}{lcccccccccccc}
\hline & \multicolumn{10}{c}{ Avaliação } \\
\hline & $\begin{array}{l}\text { Envenenamento } \\
\text { não excluído }\end{array}$ & $\begin{array}{c}\text { Envenenamento } \\
\text { leve }\end{array}$ & $\begin{array}{c}\text { Envenenamento } \\
\text { moderado }\end{array}$ & $\begin{array}{c}\text { Envenenamento } \\
\text { grave }\end{array}$ & Ignorado & Total \\
\hline Sexo & $\mathrm{N}$ & $\%$ & $\mathrm{~N}$ & $\%$ & $\mathrm{~N}$ & $\%$ & $\mathrm{~N}$ & $\%$ & $\mathrm{~N}$ & $\%$ & $\mathrm{~N}$ & $\%$ \\
Masculino & 8 & 4,7 & 33 & 19,3 & 58 & 33,9 & 62 & 36,3 & 10 & 5,8 & 171 & 100 \\
Feminino & 16 & 6,7 & 77 & 32,4 & 85 & 32,7 & 49 & 20,6 & 11 & 4,6 & 238 & 100 \\
\hline Total & 24 & 11,4 & 110 & 51,7 & 143 & 66,6 & 111 & 56,9 & 21 & 10,4 & 409 & 100 \\
\hline
\end{tabular}

$\mathrm{p}<0,001$

\section{DISCUSSÃO E CONCLUSÃO}

Analisando os resultados encontrados, percebe-se que houve uma predominância do ato suicida no sexo feminino, convergindo com a maioria dos estudos descritivos relativos às tentativas de suicídio. Segundo uma pesquisa realizada (10), após calcular a prevalência de comportamentos suicidas em uma população do município de João Pessoa-PB, verificou-se que as mulheres possuem maiores chances de ideação e planejamento suicida do que os homens, além de serem as que compõem a maior parcela da população que tenta suicídio.

Em tempo, outros estudos também evidencia a predominância da mulher na tentativa de suicídio, verificou também que as mulheres tendem a se utilizar de métodos menos efetivos de findar a própria vida, como o envenenamento; enquanto que os indivíduos do sexo masculino, apesar das tentativas serem menores, utilizam instrumentos e armas mais letais em seu intento, sendo que estes possuem maior representatividade nos casos de suicídio propriamente dito $(11,12)$.

Com relação ao fator idade, houve uma maior prevalência do número de casos de tentativa de suicídio na faixa representada pelos adultos jovens. Considerando que a ação de autodestruição é classificada como uma espécie de violência e que esta afeta principalmente o segmento populacional formado por adolescentes e adultos jovens, os resultados obtidos vão de acordo com o esperado.

O relatório da organização Mundial da Saúde (1) detectou a predominância de faixas etárias semelhantes ao deste estudo. Bem como em pesquisa realizada em Barbacena (13), dentre os 894 Boletins de Ocorrências analisados de pessoas que tentaram suicídio, $276(30,87 \%)$ são de jovens na faixa etária dos 20 aos 29 anos.

Quanto à ocupação dos pacientes atendidos por tentativa de suicídio, observou-se uma maior representatividade nas seguintes categorias: estudantes, desempregados, e a minoria que trabalhava exercia atividade de empregadas domésticas ou eram agricultores. Essas classes profissionais constituíram $44,4 \%$ do total dos casos e coincidiram com o delineado em outros estudos. No entanto, as ocupações "dona de casa" e "aposentado", que tiveram uma prevalência ínfima entre os indivíduos atendidos pelo CEATOX de Fortaleza, obtiveram uma importância significativa nos resultados de outros estudos encontrados na literatura. O que deve significar que aqui houve uma variação no padrão normalmente encontrado, ou que esses números foram subestimados devido à grande quantidade de fichas de notificação em que não 
houve o preenchimento dos dados referentes à profissão do paciente.

Ainda na pesquisa em Barbacena (13), identificou que a ocupação das pessoas que tentaram suicídio em sua maioria por: domésticas e estudantes. Assim como, outro estudo (14) identificou frequências significativas de desempregados entre os homens e donas-de-casa/aposentadas entre as mulheres.

Com relação ao local de ocorrência, a maior parte das tentativas de suicídio ocorreu em zona urbana, na própria residência do indivíduo. Pressupondo-se assim, que o local de maior acesso aos agentes tóxicos éo próprio lar do paciente. Em continuidade, segundo pesquisa realizada por Bernardes et al. (14) com pacientes de tentativa de suicídio, houve associação de outras substâncias químicas em $51,5 \%$ dos casos, com frequência maior entre os homens. Entre os fatores apontados, que favoreceram a excessiva exposição da população aos produtos químicos, estavam: desemprego, distribuição e comércio dos fármacos no país; a prescrição indiscriminada de drogas psicoativas.

Detectou-se que os agentes mais utilizados para a auto-intoxicação foram os medicamentos, sendo que destes, uma grande proporção foi composta por psicofármacos, incluindo: anticonvulsivantes, antidepressivos, ansiolíticos e antipsicóticos. O segundo grupo de agentes mais utilizados foi o dos agrotóxicos de uso agrícola, incluindo: o "chumbinho", os organofosforados e os carbamatos. Percebe-se então, que o controle ou planejamento de ações para se minimizarem a exposição da população suicida aos produtos tóxicos, devem ser voltados principalmente a essas duas classes. No caso do "chumbinho", cujo nome cientifico é ldicar$b e$, principal agrotóxico utilizado de forma irregular, como raticida doméstico (chumbinho) e dos psicotrópicos, que foram as substâncias mais utilizadas dentre os agrotóxicos e os medicamentos, respectivamente, nota-se uma falha na fiscalização de venda e dispen- sação dos mesmos, considerando que a comercialização do "chumbinho" em zona urbana é ilegal, por ser um produto clandestino e que os psicofármacos possuem um controle comercial e de dispensação específico para se evitar a utilização não médica dos mesmos. E uma vez que essas substâncias compõem grande parcela dos agentes utilizados para tentar suicídio, entende-se que houve uma facilidade de acesso da população estudada a esses tóxicos.

Outros estudos também confirmam a predominância desses dois grupos dentre as toxinas utilizadas pelos pacientes para tentativa de suicídio e identificaram que os medicamentos foram os mais utilizados nas ações de autodestruição por intoxicação, sendo responsáveis por $56 \%$ dos casos, e os praguicidas ficaram em segundo lugar com $34 \%$ das ocorrências (15). Assim como Bernardes et al. (14), que também mostrou que os medicamentos foram as substancias químicas mais utilizadas nas tentativas de suicídio, salientando inclusive que os psicofármacos representaram metade desse grupo (anticonvulsivantes, sedativos, antipsicóticos e antidepressivos).

No que se refere à quantidade de tóxicos usados para a tentativa de suicídio, ficou bastante evidenciado as ocorrências em que os pacientes utilizaram mais de uma substância ou classe de toxina. De acordo com Rosa et al. (16), o uso de vários agentes tóxicos está relacionado à maior intenção suicida, levando em consideração o conhecimento da população sobre os efeitos negativos que as interações medicamentosas podem causar.

Puderam ser identificados sete casos em que os pacientes possuíam algum transtorno mental, não sendo especificado qual. Considerando que as fichas de notificação do CEATOX de Fortaleza não possuem uma padronização para o registro de transtornos mentais voltada aos pacientes atendidos por tentativa de suicídio, esse número de ocorrência pode ter sido subestimado.

De acordo com WHO (1), os transtornos 
mentais são fatores que possuem forte relação com os casos de suicídio consumado, enquanto que nas tentativas de suicídio prevalecem os fatores relacionados com problemas afetivo-sociais. No entanto, uma investigação realizada por Santos et al. (17), encontrou um total de $71,1 \%$ de transtornos mentais entre os pacientes atendidos por tentativa de suicídio em um hospital de emergência do Rio de Janeiro. Assim como, identificaram diagnósticos psiquiátricos em $50 \%$ da população estudada (18).

$\mathrm{Na}$ presente pesquisa, quase totalidade dos indivíduos se utilizou da via oral para se auto-intoxicar, de forma que a principal conduta utilizada no tratamento destes foi a associação de lavagem gástrica com administração de carvão ativado (64,3\%). Assim, grande parte do tratamento dos pacientes atendidos por tentativa de suicídio $(98,8 \%)$ foi baseada apenas na assistência clínica, sem suporte psicológico ou psiquiátrico durante o período de internação ou encaminhamento para instituição psiquiátrica após a melhora clínica da intoxicação.

Ressalta-se, o suporte psicológico e psiquiátrico ao paciente durante a internação é de fundamental importância para prevenir futuras reincidivas de caso. Sendo assim, mesmo que, na maior parte das ocorrências deste estudo, os pacientes tenham se restabelecido, esse estado saudável concerne apenas à condição física do indivíduo. Como não houve acompanhamento psicológico ou psiquiátrico, na maioria dos casos, existem possibilidades desses pacientes voltarem a tentar suicídio. Estudo (19) corrobora com essa percepção supracitada ao afirma que quanto maior o número de tentativas, maiores são as chances de o ato ser executado com êxito.

Foi identificado que houve uma forte relação entre o grau de envenenamento dos pacientes e a variável sexo ( $\mathrm{p}<0,001)$, sendo que a maior parte da população feminina foi avaliada como estado de envenenamento moderado $(32,7 \%)$ e a maior proporção dos indivíduos do sexo masculino foi ava- liada como estado de envenenamento grave $(36,3 \%)$. Evidenciando que, mesmo na tentativa de suicídio, os homens detêm maior intencionalidade na ação suicida e/ou maior acesso a agentes mais letais. Como já citado anteriormente $(11,12)$, no qual mostram que os homens possuem maiores chances de consumar o suicídio.

Em síntese, a partir desse estudo realizado foi possível constatar a predominância do ato suicida no sexo feminino, adulto jovem, estudante, tendo o local de ocorrência a própria residência e os medicamentos foram os tóxicos mais utilizados. A principal conduta utilizada no tratamento foi a associação de lavagem gástrica com administração de carvão ativado, tendo prevalência da evolução para estabilização do quadro de intoxicação. Ao correlacionar o sexo com o nível de envenenamento, prevaleceu o nível moderado nas mulheres e grave para os homens.

Como evidenciam os dados, as ações suicidas, apesar de constituírem uma pequena parcela das causas externas, afetam significativamente as faixas etárias mais jovens e, tanto o suicídio como as tentativas de suicídio, possuem uma curva ascendente de forma que a prevalência desses agravos tende a aumentar com o passar do tempo. Diante desse problema, é visível a necessidade de maior intervenção por parte das políticas públicas.

Considera-se oportuno referir uma limitação do presente estudo ao fato dos resultados obtidos expressarem a realidade específica de um centro toxicológico, necessitando de outras pesquisas para se traçar o perfil global de pacientes que tentaram suicídio.

Assim, este estudo evidenciou resultados quanto ao perfil dos pacientes atendidos por tentativa de suicídio, no Centro de Assistência Toxicológica de um hospital de Fortaleza, Ceará, no ano de 2010, tronando-se relevante na medida em que identifica a prevalência dos determinantes e condicionantes que favorecem ou protegem o indivíduo do ato suicida, fornecendo informações essenciais para o conhecimento dessa causa externa, além 
de contribuir de forma a poder ser utilizado como guia no planejamento de políticas públicas efetivas, de prevenção e promoção da saúde, imprescindíveis para a diminuição do impacto das tentativas de suicídio na sociedade.

Consoante percebe-se, o estudo possibilitou ampliar o conhecimento sobre o perfil de pacientes que tentaram suicídio. Com base nesse conhecimento, podem-se apontar estratégias de trabalho mais condizentes com a realidade e políticas mais eficazes que busquem estimular uma maior integralidade na assistência aos indivíduos que tentam suicídio, realizando ações de promoção e prevenção, uma vez que esse agravo pode e deve ser evitado. Para tal, exige-se esforço, tanto dos profissionais de saúde como um todo - responsável por várias visões propiciadas pela clínica - quanto de gestores e familiares. É preciso proporcionar, sobretudo, um planejamento de ações frente ao controle desse agravo.

\section{REFERÊNCIAS}

1. World Health Organization. Preventing suicide: a global imperative [Internet]. Geneva: World Health Organization; 2014 [citado 10 junho 2015]. Disponível em: http://apps.who.int/iris/bitstre$\mathrm{am} / 10665 / 131056 / 8 / 9789241564878_{\text {_ }}$ eng.pdf?ua $=1 \& u a=1$

2. Durkheim E. O Suicídio: estudo de sociologia. Tradução: Mônica Stahel. São Paulo: Martins Fontes; 2000.

3. World Health Organization. Mental health: suicide prevention (Supre) [Internet]. Geneva: World Health Organization; 2012 [citado 10 junho 2015]. http://www. who.int/mental_health/prevention/suicide/suicideprevent/es/index.html

4. Mascarenhas MDM, Monteiro RA, Sá NNB, Gonzaga LAA, Neves ACM, Roza $\mathrm{DL}$, et al. Epidemiologia das causas exter- nas no Brasil: mortalidade por acidentes e violências no período de 2000 a 2009. En: Saúde Brasil 2010: uma análise da situação de saúde e de evidências selecionadas de impacto de ações de vigilância em saúde. Brasília: Ministério da Saúde; 2011: 225-50.

5. Lovisi GM, Santos AS, Lagay L, Abelha L, Valencia E. Análise epidemiológica do suicídio no Brasil entre 1980 e 2006. Rev Bras Psiquiatr. 2009; 31(Supl II): S86-93.

6. Ceará, Secretaria de Saúde do Estado. Indicadores e Dados Básicos para a Saúde no Ceará. Ceará: Secretaria de Saúde; 2007.

7. Sampaio MA, Boemer MR. Suicídio: um ensaio em busca de um des-velamento do tema. Rev Esc Enferm USP. 2000; 34(4): 325-31.

8. Bobes J, Giner J, Saiz J. Suicidio y psiquiatría. Recomendaciones preventivas y de manejo del comportamiento suicida. Madrid: Triacastela; 2011.

9. Toro GVR, Nucci NAG, Toledo TB, Oliveira AEG, Prebianchi HB. O desejo de partir: um estudo a respeito da tentativa de Suicídio. Psicologia em Revista. 2013; 19(3): 407-21.

10. Araujo LC, Vieira KFL, Coutinho MPL. Ideação suicida na adolescência: um enfoque psicossociológico no contexto do ensino médio. Psico-USF. 2010; 15(1): 47-57.

11. Pordeus AMJ, Cavalcanti LPG, Vieira LJES, Coriolano LS, Osório MM, Ponte MSR, et al. Tentativas e óbitos por suicídio no município de Independência, Ceará, Brasil. Cien Saude Colet. 2009; 14(5): 1731-40.

12. Macente LB, Santos EG, Zandonade E. Tentativas de suicídio e suicídio em município de cultura pomerana no interior do estado do Espírito Santo. J Bras Psiquiatr. 2009; 58(4): 238-44.

13. Vidal CEL, Gontijo ECDM, Lima LA. Tentativas de suicídio: fatores prognósticos e estimativa do excesso de mortalida- 
de. Cad Saude Publica. 2013; 29(1): 17587.

14. Bernardes SS, Turini CA, Matsuo T. Perfil das tentativas de suicídio por sobredose intencional de medicamentos atendidas por um Centro de Controle de Intoxicações do Paraná, Brasil. Cad Saude Publica. 2010; 26(7): 1366-72.

15. Damas FB, Zannin M, Serrano AÍ. Tentativas de suicídio com agentes tóxicos: análise estatística dos dados do CIT/SC (1994 a 2006). Revista Brasileira de Toxicologia. 2012; 25(1-2): 41-48.

16. Rosa NM, Campos APS, Guedes MRJ, Sales CCF, Mathias TAF, Oliveira MLF. Intoxicações associadas às tentativas de suicídio e suicídio em crianças e adolescentes.
Rev enferm UFPE. 2015; 9(2): 661-668.

17. Santos AS, Lovisi G, Legay L, Abelha Lúcia. Prevalência de transtornos mentais nas tentativas de suicídio em um hospital de emergência no Rio de Janeiro, Brasil. Cad Saude Publica. 2009; 25(9): 2064-74.

18. Ficher AMFT, Vansan GA. Tentativas de suicídio em jovens: aspectos epidemiológicos dos casos atendidos no setor de urgências psiquiátricas de um hospital geral universitário entre 1988 e 2004. Estud psicol (Campinas). 2008; 25(3): 361-74.

19. Souza VS, Alves MS, Silva LA, Lino DCSF, Nery AA, Casotti CA. Tentativas de suicídio e mortalidade por suicídio em um município no interior da Bahia. J bras psiquiatr. 2011; 60(4): 294-300. 